\title{
Smoking, acute mountain sickness and altitude acclimatisation: a cohort study
}

\author{
Tian-Yi Wu ${ }^{1,2,3}$ Shou-Quan Ding, ${ }^{4}$ Jin-Liang Liu, ${ }^{4}$ Jian-Hou Jia, ${ }^{4}$ Zuo-Chun Chai, ${ }^{4}$ \\ Rui-Chen Dai, ${ }^{4}$ Ji-Zhui Zhao, ${ }^{5}$ Qi De Tang, ${ }^{5}$ Bengt Kayser ${ }^{6}$
}

${ }^{1}$ Physiological Research Group of the Ministry of Railroad, Beijing, People's Republic of China

${ }^{2}$ National Key Laboratory of High Altitude Medicine, High Altitude Medical Research Institute, Qinghai, People's Republic of China

${ }^{3}$ High Altitude Medical Research Center, University of Tibet, Lhasa, Tibet, People's Republic of China

${ }^{4}$ Qingai-Tibet Railroad Hospitals of the Construction Company, Golmud, People's Republic of China

${ }^{5}$ China Railroad Construction

Company, Beijing, People's Republic of China

${ }^{6}$ Institut des sciences du mouvement et de la médecine du sport, Faculté de médicine, Université de Genève, Geneva, Switzerland

\section{Correspondence to}

Dr Tian Yi Wu, National Key Laboratory of High Altitude

Medicine, High Altitude Medical Research Institute, Xining, Zhuanchang Rd, No. 7, Qinghai 810012, People's Republic of China;

wutianyiqg@hotmail.com

Received 15 June 2011 Accepted 18 April 2012

Published Online First 14 June 2012

\section{ABSTRACT}

Rationale The relationship between cigarette smoking and acute mountain sickness (AMS) is not clear.

Objective To assess AMS risk and altitude acclimatisation in relation to smoking.

Methods 200 healthy non-smokers and 182 cigarette smokers were recruited from Han lowland workers. These were men without prior altitude exposure, matched for age, health status and occupation, who were transported to an altitude of 4525 masl.

Measurements AMS, smoking habits, arterial saturation $\left(\mathrm{SpO}_{2}\right)$, haemoglobin $(\mathrm{Hb})$, lung function and mean pulmonary artery pressure (PAPm) were assessed upon arrival and after 3 and 6 months.

Main results Compared with non-smokers, smokers had a lower incidence of AMS and lower AMS scores than non-smokers upon arrival; higher $\mathrm{Hb}$ and PAPm associated with lower $\mathrm{SpO}_{2}$ at 3 and 6 months at altitude; and lower forced expiratory volume in $1 \mathrm{~s}$ and maximal voluntary ventilation at 3 and 6 months.

Conclusions Smoking slightly decreases the risk of AMS but impairs long-term altitude acclimatisation and lung function during a prolonged stay at high altitude.

\section{INTRODUCTION}

In China in $2010,53 \%$ of men and $28 \%$ overall smoked tobacco. ${ }^{1}$ Apart from its general health risks, smoking may influence altitude hypoxia tolerance. According to some it aggravates hypoxaemia and hence increases the risk for acute mountain sickness (AMS) (Hultgren, p.469), ${ }^{2}$ but mountaineers find that smoking decreases AMS risk. ${ }^{3}$ However, sound epidemiological data on the effects of smoking on risk and disease course of AMS are lacking. During the construction of the Oinghai-Tibet railroad from 2001 to 2005, >78 000 lowland workers ascended to work and live at altitude. Since $34 \%$ of the employed Han male workforce smoked cigarettes, this presented a unique occasion to directly investigate the effects of smoking on AMS risk. We therefore recruited construction workers ascending from low altitude to work and live at the highest construction sites at an average altitude of 4552 masl. We measured AMS incidence and progression, and acclimatisation in smokers and non-smokers.

\section{METHODS}

Three hospitals participated (4779 masl, barometric pressure $(\mathrm{Pb}) \sim 417 \mathrm{~mm} \mathrm{Hg} ; 4505$ masl, $\mathrm{Pb}$ $\sim 440 \mathrm{~mm} \mathrm{Hg} ; 4292$ masl, $\mathrm{Pb} \sim 447 \mathrm{~mm} \mathrm{Hg}$ ). The

\section{Key messages}

What is the key question?

- Are smokers really better off at altitude?

What is the bottom line?

- Smokers may be better off initially at altitude, but not in the long term.

\section{Why read on?}

- This study allows us to advise smokers on altitude exposure using solid epidemiological data and suggests new avenues for research on acute mountain sickness pathophysiology.

highest work site was at 4905 masl. The study was approved by the China National Science Foundation and the Oinghai High Altitude Medical Research Institute Committee on Human Research. In 2003, a first group of 4683 workers was recruited. All prospective workers filled out a questionnaire providing information on age, sex, ethnicity, occupation, place of birth, altitude exposure, personal and family medical history, smoking and drinking behaviour. Subjects were interviewed and underwent a physical exam. Subjects in good health and physical condition were offered a job. The subjects were then asked to participate in a study on the health effects of altitude exposure. Subjects were kept unaware of the study objective, were not given information on smoking, received no incentives, were informed about procedures, knew they could withdraw at any time and gave signed consent. We sequentially recruited 200 lowland smoking and 200 non-smoking apparently healthy non-acclimatised male first-time ascenders, based on capacity. Three smokers and four non-smokers refused to participate. Groups had similar age, body mass index, working altitude and work (semimechanised, laying out tracks). Subjects travelled for 2 days by train to 2261 masl, stayed there for 2 days, and then travelled on for $12 \mathrm{~h}$ by train to 2808 masl where they stayed for 3 days. The final altitude was reached after a further $6-8 \mathrm{~h}$ bus ride.

A smoker was someone who smoked 10 or more cigarettes/day for $>6$ months. Non-smokers had never smoked; occasional smokers were excluded. Smoking was classified as mild ( $<1$ pack/day, ie, 10-20 cigarettes/day), moderate (1 pack/day) or heavy ( $>1$ pack/day). Smoking duration was short term (6 months to 2 years), medium term 
( $2-5$ years), and long term ( $>5$ years). There were only cigarette smokers.

Arterial oxygen saturation $\left(\mathrm{SpO}_{2}\right.$, finger oximetry, Ohmeda, Louisville, CO, USA) was measured in a seated position after 30 min of rest. Mean pulmonary artery pressure (PAPm) was estimated by Doppler. With a 3.5 MHz transducer (HP-Sonos 1000 or 1500, Palo-Alto, CA, USA) data were obtained from the parasternal short-axis or apical position, the subject lying in slight left oblique rotation. Recordings were stored on videotape for post hoc analysis by two independent cardiologists, unaware of smoking or altitude status. PAPm was estimated using the Kitabatake formula. In our institute correlation with directly measured pressure during right-heart catheterisation is high $\left(\mathrm{R}^{2}=0.90\right)$. PAPm $\geq 25 \mathrm{~mm} \mathrm{Hg}$ was considered pulmonary hypertension. Vital capacity (VC), forced expiratory volume in 1 s ( $\mathrm{FEV}_{1}$ ), forced expiratory flow between $25 \%$ and $75 \%$ of vital capacity $\left(\mathrm{FEF}_{25-75 \%}\right)$ and $20 \mathrm{~s}$ maximal voluntary ventilation (MVV) were measured with a portable spirometer (COSMED, Italy). Haemoglobin $(\mathrm{Hb})$ was measured on venous blood (Au-400, Olympus, Shinjuku, Tokyo, Japan). Measurements were done at low altitude, upon arrival (first hour, except PAPm, next day, and lung function, upon arrival and after 3 days), and again after 1 week, 3 months and 6 months.

AMS was assessed with Lake Louise Scoring (LLS), ${ }^{4}$ which consists of self-reported assessment of symptoms (headache, dizziness/light-headedness, fatigue, gastrointestinal upset (anorexia-nausea-vomiting) and difficulty sleeping), each scored from 0 to 3 (nil, mild, moderate, severe). It was completed with three clinical signs (change in mental status $(0-4)$, ataxia determined with heel-to-toe test (0-4), and peripheral oedema $(0-2))$. We used two cut-offs for AMS defining it as headache and a cumulative score $\geq 3$ or $\geq 4$. Severity of AMS was defined as mild for a score of $3-5$ and severe for a score of 5 or more. AMS was assessed on the evening of arrival at 4525 masl and the following evenings, for 1 week.

Data were analysed with SAS version 8.1 and are presented as mean \pm SD. Significance was set at $p<0.05$. AMS incidence was calculated as cumulative case rate. Frequencies were compared by $\chi^{2}$ test. Means were compared by t test. Pearson correlation was used for relationships between AMS scores and $\mathrm{SpO}_{2}, \mathrm{Hb}$, $\mathrm{PAPm}$, and lung function measures. Lung function changes were analysed by two-way repeated measures analysis of variance, Tuckey's post hoc test and t test for group comparisons. Crude ORs with $95 \%$ CIs were calculated to quantify the association between smoking and AMS. Univariate logistic regression

Table 1 Symptoms and signs of acute mountain sickness in nonsmokers at 4525 masl

\begin{tabular}{lrllll}
\hline & \multicolumn{5}{c}{$\mathbf{n}(\%)$} \\
\cline { 2 - 7 } LLS symptom intensity & $\mathbf{0}$ & $\mathbf{1}$ & $\mathbf{2}$ & $\mathbf{3}$ & \multicolumn{1}{c}{ Total } \\
\hline Headache & $46(23)$ & $70(35)$ & $56(28)$ & $28(14)$ & $154(77)$ \\
Dizziness or light-headedness & $158(79)$ & $22(11)$ & $12(6)$ & $8(4)$ & $42(21)$ \\
Weakness or fatigue & $87(43)$ & $68(34)$ & $42(21)$ & $3(2)$ & $113(57)$ \\
Anorexia, nausea or vomiting & $102(51)$ & $52(26)$ & $36(18)$ & $10(5)$ & $98(49)$ \\
Difficulty sleeping & $58(29)$ & $72(36)$ & $58(29)$ & $12(6)$ & $142(71)$ \\
Reduction in activity & $112(56)$ & $71(36)$ & $17(9)$ & 0 & $88(44)$ \\
Change in mental status & $196(98)$ & $3(1.5)$ & $1(0.5)$ & 0 & $4(2)$ \\
Ataxia & $194(97)$ & $6(3)$ & 0 & 0 & $6(3)$ \\
Peripheral oedema & $172(86)$ & $21(11)$ & $7(3)$ & 0 & $28(14)$ \\
\hline
\end{tabular}

Comparison between control group and smoking group for headache, $\chi^{2}=4.66, p=0.031$; for anorexia, nausea or vomiting, $\chi^{2}=3.85, p=0.049$; for difficulty sleeping $\chi^{2}=13.517$, $\mathrm{p}<0.001$; for all other symptoms differences were non-significant. Total: the sum of scores $>0$. analysis was used to estimate AMS risk for smoking versus control and to examine relationships between individual variables and presence of AMS. Multiple logistic regression analysis was performed to test for the effects of independent variables and identify the main effects. Significant risk factors were entered into forward regression using the likelihood ratio test. The dichotomous dependent variable was AMS $(0=$ no AMS, $1=$ AMS, LLS cutoff score $\geq 4$ ). Independent variables were $\mathrm{SpO}_{2}, \mathrm{Hb}, \mathrm{PAPm}, \mathrm{VC}, \mathrm{FEV}_{1}, \mathrm{FEF}_{25-75 \%}$ and $\mathrm{MVV}$. $\mathrm{SpO}_{2}$ was recoded into $0=$ at least $90 \%, 1=86-89 \%$ and $2=$ up to $85 \%$. $\mathrm{Hb}$ concentration was recoded into $0=$ up to $16 \mathrm{~g} / \mathrm{dl}$ and $1=$ greater than $16 \mathrm{~g} / \mathrm{dl}$. PAPm was recoded into $0=$ up to $20 \mathrm{~mm} \mathrm{Hg}$ and $1=$ greater than $20 \mathrm{~mm} \mathrm{Hg}$. VC, $\mathrm{FEV}_{1}, \mathrm{FEF}_{25-75 \%}$ and $\mathrm{MVV}$ was recoded as $0=$ normal low altitude value and $1=$ abnormal, that is, increased or decreased by $>2 \mathrm{SD}$ from the low altitude value. Smoking behaviour was coded as $0=$ no smoking, $1=$ less than 1 pack/day, $2=$ about 1 pack/day and $3=$ more than 1 pack/day. Smoking history was coded as $0=$ no smoking, $1=$ short-tem, $2=$ medium-term and $3=$ long-term smoking.

\section{RESULTS}

\section{Subjects}

Four smokers withdrew before ascent and 14 were lost to followup at altitude for non-medical reasons; all non-smokers completed the study. We obtained data from 182 smokers (SMO, age $38 \pm 7$ years, range 25-54 years) and 200 non-smokers (CON, $38 \pm 6$ years, $24-56$ years).

\section{Smoking}

The SMO group comprised $18 \%$ mild, $45 \%$ moderate and $37 \%$ heavy smokers. Smoking habit was 23\% short term, 35\% medium term and $42 \%$ long term. At high altitude, packs/day smoked remained similar to low-altitude smoking ( $p>0.05)$.

\section{Acute mountain sickness}

AMS incidence in SMO was lower than in CON (LLS $\geq 3: 45 \%$ vs $56 \%, \chi^{2}=4.57, \mathrm{p}=0.039 ; \mathrm{LLS} \geq 4: 39 \%$ vs $51 \%, \chi^{2}=5.53, \mathrm{p}=0.013$; LLS $\geq 5$ : $3.4 \%$ vs $8.5 \%, \chi^{2}=4.56, p=0.038$ ). Five per cent of subjects with LLS $\geq 5$ were hospitalised, more from CON than from SMO ( 17 vs 6 cases, $\chi^{2}=4.56, p=0.038$ ). On arrival, SMO had a lower LLS score than CON (1.6 \pm 0.6 vs $1.8 \pm 0.7, p=0.004)$. SMO with $L L S \geq 3$ had lower scores than CON $(3.8 \pm 0.5$ vs $4.0 \pm 0.6, \mathrm{p}<0.001)$. At 1 week SMO still had lower scores than CON (1.4 \pm 0.8 vs $1.6 \pm 0.5, p=0.005)$. There was no altitude cerebral or pulmonary oedema. $\mathrm{SpO}_{2}$ correlated negatively with LLS score (CON: $R=-0.192, p=0.005$; SMO: $R=-0.174$, $\mathrm{p}=0.019$; no difference between groups, $\mathrm{p}=0.095)$. PAPm

Table 2 Symptoms and signs of acute mountain sickness in smokers at 4525 masl

\begin{tabular}{lrlllc}
\hline & \multicolumn{5}{c}{$\mathbf{n ~ ( \% )}$} \\
\cline { 2 - 6 } LLS symptom intensity & $\mathbf{0}$ & $\mathbf{1}$ & $\mathbf{2}$ & $\mathbf{3}$ & Total \\
\hline Headache & $104(57)$ & $42(23)$ & $22(12)$ & $14(8)$ & $78(43)$ \\
Dizziness or light-headedness & $144(79)$ & $22(12)$ & $13(7)$ & $3(2)$ & $38(21)$ \\
Weakness or fatigue & $81(45)$ & $50(27)$ & $42(23)$ & $6(3)$ & $101(55)$ \\
Anorexia, nausea or vomiting & $111(61)$ & $39(21)$ & $26(14)$ & $6(3)$ & $71(39)$ \\
Difficulty sleeping & $86(47)$ & $42(23)$ & $45(25)$ & $9(5)$ & $96(53)$ \\
Reduction in activity & $107(59)$ & $58(32)$ & $15(8)$ & $2(1)$ & $75(41)$ \\
Change in mental status & $180(99)$ & $2(1)$ & 0 & 0 & $2(1)$ \\
Ataxia & $178(98)$ & $4(2)$ & 0 & 0 & $4(2)$ \\
Peripheral oedema & $158(87)$ & $18(10)$ & $6(3)$ & 0 & $24(13)$ \\
\hline
\end{tabular}

See table 1. 
Table 3 Mean $( \pm S D)$ peak scores of Lake Louise Scoring symptoms

\begin{tabular}{lllr}
\hline Symptom & CON & SM0 & p Value \\
\hline Headache & $1.33 \pm 0.56$ & $0.70 \pm 0.42$ & $<0.001$ \\
Dizziness or light-headedness & $0.35 \pm 0.30$ & $0.31 \pm 0.18$ & 0.192 \\
Weakness or fatigue & $0.88 \pm 0.26$ & $0.89 \pm 0.28$ & 0.755 \\
Anorexia, nausea or vomiting & $0.77 \pm 0.42$ & $0.60 \pm 0.36$ & $<0.001$ \\
Difficulty sleeping & $1.48 \pm 0.45$ & $0.95 \pm 0.38$ & $<0.001$ \\
\hline
\end{tabular}

CON, control group; SMO, smoking group.

correlated negatively with LLS score (CON: $R=-0.147$, $\mathrm{p}=0.044$; SMO: $\mathrm{R}=-0.156, \mathrm{p}=0.048$; no difference between groups $\mathrm{p}=0.075)$. There were no significant correlations with other variables. SMO suffered less from headache, anorexianausea-vomiting or sleep disturbances than CON but reported similar frequency and intensity for the other LLS symptoms (tables 1 and 2). Average peak scores for separate AMS symptoms differed significantly for headache, anorexianausea-vomiting and difficulty sleeping (table 3).

\section{Lung function}

On arrival at 4525 masl VC tended to be lower in both groups (table 4). On day 3 the mean decrease was $4 \%$ and $6 \%$ in SMO and CON respectively. VC had normalised after 3 and 6 months in $\mathrm{CON}$, but not in $\mathrm{SMO} \mathrm{FEV}_{1}$ and $\mathrm{FEF}_{25-75 \%}$ were increased in $\mathrm{CON}$ and $\mathrm{SMO}$ upon arrival. They remained higher in $\mathrm{CON}$, whereas they decreased in SMO over time. A similar pattern was observed for MVV.

\section{Oxygen saturation}

Low-altitude $\mathrm{SpO}_{2}$ values were similar (CON: 97 $\pm 7 \%$, SMO: $97 \pm 6 \%, \mathrm{p}=0.816$ ). Upon arrival, $\mathrm{SpO}_{2}$ was lower (CON: $83 \pm 6 \%, \mathrm{SMO}: 83 \pm 5 \%, \mathrm{p}=0.001$ vs low altitude, no difference between groups, $p=0.164)$. With time spent at altitude, SMO developed a lower $\mathrm{SpO}_{2}$ than $\mathrm{CON}$ (3 months: $85 \pm 5 \%$ vs $86 \pm 6 \%, p=0.004 ; 6$ months: $85 \pm 6 \%$ vs $86 \pm 6 \%, p=0.002$, table 5). This difference was due to improvement of $\mathrm{SpO}_{2}$ in $\mathrm{CON}$ by $3.8 \%$ and $4.1 \%$ after 3 and 6 months respectively, whereas SMO $\mathrm{SpO}_{2}$ only increased by $2.8 \%$ and $2.5 \%$ at 3 and 6 months respectively $(p=0.035$ and $p=0.002)$.

\section{Haemoglobin concentration}

Initially both groups had similar $\mathrm{Hb}$ (low altitude, $\mathrm{SMO}$ : $15.8 \pm 2.1 \mathrm{~g} / \mathrm{dl}, \mathrm{CON}: 15.5 \pm 1.4 \mathrm{~g} / \mathrm{dl}, \mathrm{p}=0.164$; on arrival, $\mathrm{SMO}$ : $16.0 \pm 1.8 \mathrm{~g} / \mathrm{dl}, \mathrm{CON}: 15.8 \pm 1.6 \mathrm{~g} / \mathrm{dl}, \mathrm{p}=0.189)$. After 3 months the groups differed (SMO: $16.2 \pm 1.8 \mathrm{~g} / \mathrm{dl}, \mathrm{CON}: 15.8 \pm 1.5 \mathrm{~g} / \mathrm{dl}$, $\mathrm{p}=0.021$ ). This difference was more marked after 6 months
(SMO: $17.4 \pm 1.6 \mathrm{~g} / \mathrm{dl}, \quad \mathrm{CON}: \quad 16.2 \pm 1.5 \mathrm{~g} / \mathrm{dl}, \quad \mathrm{p}<0.001$, see table 5$). \mathrm{Hb}$ increased with packs/day $(\mathrm{R}=0.22, \mathrm{p}=0.005)$ and years of smoking $(\mathrm{R}=0.23, \mathrm{p}<0.001)$. At 6 months, $\mathrm{Hb}$ was higher in heavy and long-term smokers $(17.2 \pm 2.1 \mathrm{~g} / \mathrm{dl}$ and $18.1 \pm 2.3 \mathrm{~g} / \mathrm{dl}$ respectively) than in mild and moderate smokers (crude OR 1.1, 95\% CI 1.01 to $1.26, \mathrm{p}=0.035$ ) as well as shortterm or medium-term smokers (crude OR 1.1, 95\% CI 1.11 to 1.87, $\mathrm{p}=0.011$, see table 6).

\section{Pulmonary artery pressure}

At low altitude PAPm was similar (SMO: 15.6 $\pm 3.1 \mathrm{~mm} \mathrm{Hg}$, CON: $15.1 \pm 2.8 \mathrm{~mm} \mathrm{Hg}, p=0.101)$. Both groups increased PAPm upon arrival and SMO had higher PAPm than CON $(17.5 \pm 4.5 \mathrm{~mm} \mathrm{Hg}$ vs $16.2 \pm 3.6 \mathrm{~mm} \mathrm{Hg}, \mathrm{p}=0.005)$. Over time PAPm increased further (3 months, SMO: $22.4 \pm 4.4 \mathrm{~mm} \mathrm{Hg}$, CON: $21.5 \pm 3.8 \mathrm{~mm} \mathrm{Hg}, \quad p=0.005 ; 6$ months, SMO: $23.1 \pm 4.8 \mathrm{~mm} \mathrm{Hg}, \mathrm{CON}: 21.7 \pm 4.1 \mathrm{~mm} \mathrm{Hg}, \mathrm{p}=0.023$, table 6). PAPm correlated with packs/day $(R=0.17, p=0.008)$ and years smoking $(R=0.19, p=0.005)$. At 6 months, PAPm in heavy and long-term smokers was $24.2 \pm 5.2 \mathrm{~mm} \mathrm{Hg}$ and $24.0 \pm 5.7 \mathrm{~mm} \mathrm{Hg}$ respectively, significantly higher than that of mild or moderate smokers (crude OR 1.1, 95\% CI 1.05 to 1.68 , $\mathrm{p}=0.048$ ) and short-term and medium-term smokers (crude OR $1.2,95 \%$ CI 1.01 to $1.71, \mathrm{p}=0.031$, see table 5). $\mathrm{SpO}_{2}$ correlated positively with PAPm in $\mathrm{CON}(\mathrm{R}=0.158, \mathrm{p}=0.019)$ and negatively in SMO $(R=-0.163, p=0.023)$.

\section{Logistic regression}

At altitude, subjects with $\mathrm{SpO}_{2} \leq 85 \%$ were 2.6 times more likely to have AMS than those with $\mathrm{SpO}_{2} \geq 90 \%$ (table 7). $\mathrm{Hb}$, PAPm, and lung function variables did not show significant effects. Crude ORs of $\mathrm{FEV}_{1}, \mathrm{FEF}_{25-75 \%}$ and $\mathrm{MVV}$ were similar to those of VC (not shown). Heavy smoking and medium-term or long-term smoking history decreased AMS risk (table 7). In multivariate logistic regression only $\mathrm{SpO}_{2}$, smoking habits and smoking history had significant effects (table 8).

\section{DISCUSSION \\ Acute mountain sickness}

We found an $11-12 \%$ (20-24\% relative) lower incidence of AMS for LLS cut-off scores $\geq 3$ and $\geq 4$ respectively in smokers compared with non-smokers. This contrasts with studies on AMS risk in tourists and climbers, ${ }^{5}{ }^{6}$ but confirms a tendency found in a prospective cohort study (crude OR 0.66 , 95\% CI 0.41 to $1.07, \mathrm{p}=0.09$ ). ${ }^{7}$ Hultgren $^{2}$ (p. 469) hypothesised that smokers would have more AMS and have problems acclimatising because

Table 4 Pulmonary function for SMO versus CON

\begin{tabular}{|c|c|c|c|c|c|c|c|}
\hline Parameters & Low altitude & After arrival & Day 3 & 3 months & 6 months & $\mathbf{P}^{\mathbf{a}}$ & $\mathbf{P}^{\mathbf{b}}$ \\
\hline \multirow[t]{2}{*}{ VC (I) } & SM0 $4.48 \pm 0.46$ & SM0 $4.36 \pm 0.63$ & SM0 $4.24 \pm 0.60$ & SM0 $4.12 \pm 0.62$ & SM0 $4.10 \pm 0.38$ & 0.046 & G: 0.110 \\
\hline & $\operatorname{CON} 4.54 \pm 0.44$ & $\operatorname{CON} 4.43 \pm 0.45$ & $\operatorname{CON} 4.12 \pm 0.63$ & $\operatorname{coN} 4.52 \pm 0.32^{*}$ & $\operatorname{coN} 4.50 \pm 0.26 \dagger$ & 0.013 & I: 0.027 \\
\hline $\mathrm{FEV}_{1}$ (I) & SM0 $3.92 \pm 0.82$ & SM0 $3.98 \pm 0.74$ & SM0 $4.02 \pm 0.84$ & SM0 $3.96 \pm 0.78$ & SM0 $3.82 \pm 0.74$ & 0.321 & G: 0.164 \\
\hline \multirow[t]{2}{*}{$\mathrm{FEF}_{25-75 \%}$ (litres/s) } & SM0 $4.08 \pm 1.05$ & SM0 $4.01 \pm 0.85$ & SM0 $4.06 \pm 1.06$ & SM0 $4.02 \pm 1.12$ & SM0 $3.92 \pm 0.92$ & 0.465 & G: 0.044 \\
\hline & CON $4.16 \pm 0.85$ & $\operatorname{CON} 4.24 \pm 0.66$ & $\operatorname{CON} 4.28 \pm 0.72$ & CON $4.81 \pm 0.63 \S$ & $\operatorname{CON} 4.93 \pm 0.679$ & 0.006 & I: 0.048 \\
\hline MVV (litres/min) & SM0 $108.0 \pm 4.5$ & SMO $110.2 \pm 4.4$ & SMO $111.6 \pm 4.8$ & SMO $106.3 \pm 5.2$ & SM0 107.4 \pm 5.3 & 0.035 & G: 0.002 \\
\hline
\end{tabular}

Data are presented as mean \pm SD. $P^{a}$ : ANOVA for repeated measures within each group separately. $\mathrm{P}^{\mathrm{b}}$ : ANOVA-probabilities between groups (G), and interaction (I). Group comparisons:

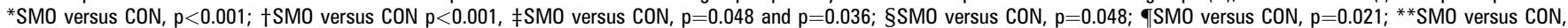
$\mathrm{p}<0.001$.

Because of technical problems only a subset of subjects had pulmonary function tests: at low altitude 40 (SMO) and 42 (CON), after arrival 36 (SMO) and 28 (CON), at 3 days 36 (SMO) and 28 (CON), at 3 months 32 (SMO) and 34 (CON) and at 6 months 28 (SMO) and 25 (CON). The measurements reported concern the same subjects over time.

SMO, smoking group; CON, control group; VC, vital capacity; FEV , forced expiratory volume in $1 \mathrm{~s}$; FEF $25-75 \%$ forced expiratory flow between $25 \%$ and $75 \%$ of vital capacity; MVV, maximal voluntary ventilation. 
Table 5 0xygen saturation, haemoglobin concentration and mean pulmonary artery pressure

\begin{tabular}{|c|c|c|c|c|}
\hline & $\mathbf{N}$ & $\mathrm{SpO}_{2}(\%)$ & $\mathrm{Hb}(\mathrm{g} / \mathrm{dl})$ & PAPm (mm Hg) \\
\hline \multicolumn{5}{|c|}{ Low altitude } \\
\hline SMO & 182 & $96.5 \pm 6.4$ & $15.8 \pm 2.1$ & $15.6 \pm 3.1$ \\
\hline CON & 200 & $97.2 \pm 6.8$ & $15.5 \pm 1.4$ & $15.1 \pm 2.8$ \\
\hline \multicolumn{5}{|c|}{ After arrival at altitude } \\
\hline SMO & 182 & $82.5 \pm 5.2$ & $16.0 \pm 1.8$ & $17.5 \pm 4.5$ \\
\hline CON & 200 & $83.2 \pm 5.8$ & $15.8 \pm 1.6$ & $16.2 \pm 3.6$ \\
\hline \multicolumn{5}{|c|}{ After 3 months } \\
\hline SMO & 182 & $84.8 \pm 4.6^{*}$ & $16.2 \pm 1.8 \neq$ & $22.4 \pm 4.49$ \\
\hline CON & 200 & $86.4 \pm 5.7$ & $15.8 \pm 1.5$ & $21.5 \pm 3.8$ \\
\hline \multicolumn{5}{|c|}{ After 6 months } \\
\hline SMO & 182 & $84.6 \pm 6.3^{*}$ & $17.4 \pm 1.6 \S$ & $23.1 \pm 4.8^{* *}$ \\
\hline CON & 200 & $86.6 \pm 5.7$ & $16.2 \pm 1.5$ & $21.7 \pm 4.1$ \\
\hline
\end{tabular}

Data are presented as mean \pm SD.

*SMO versus CON: $p=0.004$

†SMO versus CON: $p=0.001$.

$\ddagger S M 0$ versus $C O N$ : $p=0.021$.

§SMO versus CON: $p<0.001$.

TSMO versus CON: $p=0.044$

${ }^{* *}$ SMO versus CON: $p=0.004$.

$\mathrm{SMO}$, smoking group; $\mathrm{CON}$, control group; $\mathrm{Hb}$, haemoglobin concentration; PAPm, mean pulmonary artery pressure; $\mathrm{SpO}_{2}$, arterial oxygen saturation.

of aggravated hypoxaemia through diminished blood oxygencarrying capacity from carboxyhaemoglobin $(\mathrm{COHb})$, decreased oxygen uptake due to the respiratory effects of smoking, and impaired peripheral oxygen extraction. One study reported that a combination of smoking and alcohol impeded altitude acclimatisation to 3200 masl in lowland workers, but did not report AMS. $^{8}$

\section{Headache, gastrointestinal upset and sleep disturbance}

Differences in AMS incidence and severity were small but statistically highly significant. Of limited clinical relevance they are of interest for AMS pathophysiology. Smokers had less headache, anorexia-nausea-vomiting and sleep disturbance. AMS headache may result from hypoxia-induced cerebral vasodilatation or its effectors, such as nitric oxide (NO), perhaps through activation of the trigeminovascular system ${ }^{9}$ and cerebral venous hypertension. ${ }^{10}$ At low altitude $\mathrm{NO}$ plays a role in tension type headache and $\mathrm{NO}$ prodrugs are associated with headache and nausea. ${ }^{11}$ Nitroglycerin causes headache and exacerbates $\mathrm{AMS}^{12}$ as does sildenafil. ${ }^{13}$ Smoking impairs endothelial function, decreasing $\mathrm{NO}$ formation and increasing $\mathrm{NO}$ degradation ${ }^{14}$ and smokers expire less NO. ${ }^{15}$ We speculate that decreased NO levels protected smokers somewhat from headache and gastrointestinal upset.

Smokers reported fewer sleep problems. Altitude exposure induces a periodic breathing pattern. ${ }^{16}$ The oscillations result from high ventilatory sensitivity to carbon dioxide $\left(\mathrm{CO}_{2}\right)$ and hypoxia in the presence of narrowed $\mathrm{CO}_{2}$ reserve and induce frequent arousals from sleep. Nicotine, $\mathrm{NO}$ and carbon monoxide (CO) influence the regulation of breathing. ${ }^{17}{ }^{18} \mathrm{We}$ speculate that smokers slept better because of less breathing instability through higher nicotine and $\mathrm{CO}$, and lower $\mathrm{NO}$ levels.

\section{Pulmonary arterial pressure}

Smokers tended to have higher PAPm at low altitude, which is expected since smoking increases PAP. ${ }^{19}$ At altitude, both groups had increased PAPm, which was expected since hypoxia increases PAPm. ${ }^{20}$ Smokers had higher PAPm, with a small significant difference between smokers and non-smokers at 3 and 6 months, which was more pronounced in heavy smokers,
Table 6 Oxygen saturation, haemoglobin concentration and mean pulmonary artery pressure at 6 months and intensity/history of smoking

\begin{tabular}{lllll}
\hline $\begin{array}{l}\text { Smoking intensity/ } \\
\text { smoking history }\end{array}$ & $\mathbf{n}$ & $\mathbf{S p 0}_{\mathbf{2}}$ & $\mathbf{H b}(\mathbf{g} / \mathbf{d l})$ & $\begin{array}{l}\mathbf{P A P m} \\
(\mathbf{m m ~ H g})\end{array}$ \\
\hline Mild & 33 & $84.8 \pm 1.7$ & $16.3 \pm 1.6$ & $22.5 \pm 3.6$ \\
Moderate & 82 & $84.2 \pm 2.2$ & $16.5 \pm 1.7$ & $22.8 \pm 4.4$ \\
Heavy & 67 & $83.1 \pm 2.5^{*}$ & $17.2 \pm 2.1 \neq$ & $24.2 \pm 5.2 \mathbf{9}$ \\
Short term & 41 & $85.2 \pm 2.4$ & $16.6 \pm 1.6$ & $22.1 \pm 3.5$ \\
Medium term & 65 & $84.7 \pm 1.6$ & $17.2 \pm 1.8$ & $22.5 \pm 4.5$ \\
Long term & 76 & $84.2 \pm 2.1 \dagger$ & $18.1 \pm 2.3 \S$ & $24.0 \pm 5.7^{* *}$ \\
\hline
\end{tabular}

Data are presented as mean \pm SD.

*Heavy levels of smoking versus moderate $(p=0.002)$ and mild levels of smoking $(\mathrm{p}<0.001)$.

tLong-term smoking versus medium-term $(p=0.009)$ and short-term smoking $(p<0.001)$ $\ddagger$ Heavy levels of smoking versus moderate $(p=0.036)$ and mild levels of smoking $(\mathrm{p}<0.001)$.

$\S$ Long-term smoking versus medium-term $(p=0.008)$ and short-term smoking $(p<0.001)$. ॠHeavy levels of smoking versus moderate $(p=0.048)$ and mild levels of smoking $(p=0.040)$.

** Long-term smoking versus medium-term $(p=0.057)$ and short-term smoking $(p=0.044)$ $\mathrm{Hb}$, haemoglobin concentration; PAPm, mean pulmonary artery pressure; $\mathrm{SpO}_{2}$, arterial oxygen saturation.

suggesting a dose-response effect. Increased PAP at altitude is associated with high altitude pulmonary oedema (HAPE) ${ }^{21}$ People prone to HAPE exhale less NO. PAP can be lowered by inhaling NO, and increasing $\mathrm{NO}$ with tadalfil prevents HAPE. ${ }^{21}$ Since smoking impairs NO bioavailability ${ }^{14} 19$ and lowers exhaled NO levels ${ }^{15}$ we explain our findings of higher PAPm in smokers in part from decreased $\mathrm{NO}$ bioavailability in the pulmonary circulation.

\section{Saturation}

Increased $\mathrm{SpO}_{2}$ with time in non-smokers reflects ventilatory acclimatisation to altitude. ${ }^{22}$ At low arterial oxygen pressure $\left(\mathrm{PaO}_{2}\right)$ peripheral chemoreceptor activation induces hyperventilation. The sensitivity of this pathway increases with time. ${ }^{22}$ Smokers showed less increase in $\mathrm{SpO}_{2}$ at 3 and 6 months. This suggests that smoking hampers ventilatory acclimatisation to

Table 7 Results of multiple univariate regression analysis (unadjusted) for the variables in the left column

\begin{tabular}{|c|c|c|c|}
\hline Variables & AMS, n (\%) & Crude OR $(95 \% \mathrm{Cl})$ & p Value \\
\hline \multicolumn{4}{|l|}{$\mathrm{SpO}_{2}(\%)$} \\
\hline$\geq 90$ & $32(33)$ & 1 (ref) & \\
\hline $86-89$ & $65(40)$ & $0.986(0.647$ to 1.545$)$ & 0.069 \\
\hline$\leq 85$ & $76(62)$ & $2.630(2.156$ to 3.274$)$ & $<0.001$ \\
\hline \multicolumn{4}{|l|}{$\mathrm{Hb}(\mathrm{g} / \mathrm{dl})$} \\
\hline$\leq 16$ & $142(46)$ & 1 (ref) & \\
\hline$>16$ & $31(42)$ & 0.745 (0.504 to 0.762$)$ & 0.238 \\
\hline \multicolumn{4}{|l|}{ PAPm $(\mathrm{mm} \mathrm{Hg})$} \\
\hline$\leq 20$ & $164(45)$ & 1 (ref) & \\
\hline$>20$ & $9(45)$ & 0.645 (0.446 to 0.672$)$ & 0.164 \\
\hline \multicolumn{4}{|l|}{ vc } \\
\hline Normal & $168(46)$ & 1 (ref) & \\
\hline Less by $\geq 2$ SD & $5(39)$ & 0.211 (0.096 to 0.747$)$ & 0.770 \\
\hline \multicolumn{4}{|l|}{ Smoking } \\
\hline No smoking & $102(51)$ & 1 (ref) & \\
\hline$<1$ pack/day & $16(49)$ & $0.860(0.674$ to 0.901$)$ & 0.755 \\
\hline 1 pack/day & $30(37)$ & $0.786(0.652$ to 0.810$)$ & 0.035 \\
\hline$>1$ pack/day & $25(37)$ & $0.627(0.335$ to 0.856$)$ & 0.039 \\
\hline \multicolumn{4}{|l|}{ Smoking history } \\
\hline No smoking & $102(51)$ & 1 (ref) & \\
\hline Short term & $17(40)$ & $0.864(0.520$ to 0.978$)$ & 0.465 \\
\hline Medium term & $25(39)$ & $0.818(0.465$ to 1.075$)$ & 0.044 \\
\hline Long term & $29(38)$ & 0.654 (0.358 to 0.861$)$ & 0.027 \\
\hline
\end{tabular}


Table 8 Results of multiple logistic regression analysis (adjusted) with the variables in the left column retained in the final regression (all other variables not significant)

\begin{tabular}{|c|c|c|c|c|c|}
\hline Variables & $\beta$ & SE & Wald & Adjusted OR (95\% CI) & p Value \\
\hline \multicolumn{6}{|l|}{$\mathrm{SpO}_{2} \leq 85 \%$} \\
\hline Non-smoking & -0.048 & 0.481 & 14.12 & $2.343(2.121$ to 2.895$)$ & 0.001 \\
\hline Smoking & -0.062 & 0.474 & 15.86 & $2.584(1.984$ to 3.365$)$ & 0.001 \\
\hline \multicolumn{6}{|l|}{ Smoking } \\
\hline \multicolumn{6}{|l|}{ No smoking } \\
\hline$<1$ pack/day & 0.084 & 0.382 & 3.84 & $0.865(0.583$ to 1.014$)$ & 0.075 \\
\hline 1 pack/day & -0.051 & 0.257 & 4.17 & 0.662 (0.424 to 0.898 ) & 0.031 \\
\hline$>1$ pack/day & -0.056 & 0.236 & 4.28 & $0.646(0.328$ to 0.856$)$ & 0.020 \\
\hline \multicolumn{6}{|l|}{ Smoking history } \\
\hline \multicolumn{6}{|l|}{ No smoking } \\
\hline Short term & -0.086 & 0.408 & 3.86 & 0.748 (0.482 to 1.083$)$ & 0.057 \\
\hline Medium term & -0.068 & 0.252 & 4.04 & $0.674(0.412$ to 0.767$)$ & 0.027 \\
\hline Long term & -0.082 & 0.186 & 4.16 & $0.636(0.318$ to 0.825$)$ & 0.021 \\
\hline
\end{tabular}

$\mathrm{SpO}_{2}$, arterial oxygen saturation.

high altitude. Chemoreceptor function is modulated by $\mathrm{NO}$ and CO. ${ }^{17}$ Nicotine increases peripheral chemoreflex sensitivity to reductions in arterial oxygen content in non-smokers but not in smokers. ${ }^{18}$ In people who live at altitude all their lives, a decrease in ventilation may eventually develop. ${ }^{23}$ The reduced ventilatory drive results from less sensitivity of central chemoreceptors for $\mathrm{CO}_{2}$ and of peripheral chemoreceptors for hypoxia, and leads to polycythaemia. ${ }^{23}$ Since smoking is a risk factor for this syndrome $e^{24}$ we speculate that smokers showed reduced ventilatory acclimatisation from reduced chemoreceptor sensitivity.

Most oximeters, including ours, interpret carboxyhaemoglogin as $\mathrm{O}_{2}$ saturation of $\mathrm{Hb}\left(\mathrm{HbO}_{2}\right)$ and thus indicate an erroneously high $\mathrm{SpO}_{2}$ in smokers. ${ }^{25}$ Since at altitude alveolar oxygen pressure $\left(\mathrm{P}_{\mathrm{A}} \mathrm{O}_{2}\right)$ and $\mathrm{PaO}_{2}$ decrease while alveolar carbon dioxide pressure $\left(\mathrm{P}_{\mathrm{A}} \mathrm{CO}\right.$ ) remains similar (assuming $\mathrm{CO}$ exposure from smoking invariable), competition between $\mathrm{CO}$ and $\mathrm{O}_{2}$ increases $\mathrm{COHb}^{26}$ Since increased $\mathrm{COHb}$ in smokers displaces the $\mathrm{HbO}_{2}$ dissociation curve leftward, smokers likely had lower $\mathrm{PaO}_{2}$, in line with reduced peripheral chemoreceptor sensitivity in smokers. Brewer et $a^{26}$ indeed found lower $\mathrm{PaO}_{2}$ in smokers at 3100 masl than in non-smokers $(53.4 \pm 5.8 \mathrm{~mm} \mathrm{Hg}$ vs $58.6 \pm 4.2 \mathrm{~mm} \mathrm{Hg}$ ).

\section{Smoking polycythaemia}

Smoking causes polycythaemia. The tendency for higher $\mathrm{Hb}$ in smokers at low altitude became significant at 3 and 6 months at altitude. This increased blood oxygen carrying capacity, correcting for decreased saturation, as previously reported. ${ }^{26}$ Smokinginduced and hypoxia-induced erythropoiesis increased $\mathrm{Hb}$ more in smokers, placing them at higher risk of developing chronic mountain sickness if they remained at altitude for years. ${ }^{23} 24$

\section{Lung function}

VC tended to decrease upon arrival at altitude and normalised with time in non-smokers but not in smokers. Previous studies reported a decrease in VC during the first 12-24 h of altitude exposure. $^{27}$ This fall may be caused by increased pulmonary blood volume and mild interstitial oedema. ${ }^{28} \mathrm{VC}$ changes upon arrival were not related to $\mathrm{SpO}_{2}$, but subjects with AMS had greater decreases in VC than those without $(4.0 \pm 0.7$ vs $4.2 \pm 0.5 \%, \mathrm{p}<0.002)$.

Since air density decreases with altitude, increases in $\mathrm{FEV}_{1}$ and $\mathrm{FEF}_{25-75 \%}$ were expected. But data in the literature are conflicting. $\mathrm{FEV}_{1}$ was found to increase, ${ }^{29}$ decrease $^{30}$ or remain unchanged at altitude. ${ }^{31}$ After arrival at altitude, FEV $_{1}$ was slightly higher in smokers and non-smokers. This increase persisted over time in non-smokers but had decreased after 6 months in smokers, suggesting a decrease in lung function from smoking. $\mathrm{FEF}_{25-75 \%}$ was increased in non-smokers to $115 \%$ and $118 \%$ of low altitude values at 3 and 6 months, respectively. In smokers $\mathrm{FEF}_{25-75 \%}$ was similar to low altitude at 3 months and had decreased by $4 \%$ at 6 months. As expected, MVV increased in non-smokers and remained elevated at 3 months and 6 months. By contrast, MVV decreased throughout the altitude stay in smokers and overall smokers showed loss of lung function while at altitude.

\section{Strengths and limitations}

The main strength of our study is the inclusion of almost 200 smokers, allowing effects to be identified that were previously undiscovered. We did not measure exhaled levels of CO and NO, or blood gases or $\mathrm{COHb}$ levels, to relate these to AMS symptom scores. Sleep quality measured with actimetry, and quantification of the ventilatory response to hypoxia and hypercapnia might have provided further insight too. Since smoking was reported to reduce pain perception, we cannot fully exclude the fact that the perception of severity of symptoms of AMS was less in smokers compared with non-smokers. ${ }^{32}$

\section{Smoking and health}

Presenting 'positive' effects of smoking is uncomfortable; smoking must be strongly discouraged. We do not recommend smoking to prevent AMS. First, we did not study the effects in non-smokers but investigated habitual smokers. Second, smoking is strongly addictive and increases the risk of cardiorespiratory and other diseases, including cancer. ${ }^{33}$ Third, altitude is accompanied by cold exposure and smoking increases the risk of frostbite. ${ }^{34}$ Fourth, smoking decreases exercise capacity. ${ }^{35}$ Fifth, smoking represents risk for others because of secondhand smoke. ${ }^{33}$ And finally, the effect on AMS risk and severity was small. Gradual ascent and sufficient time for acclimatisation are best for AMS prevention. ${ }^{9}$

\section{CONCLUSION}

We found that non-acclimatised smokers are at slightly reduced risk for AMS at altitude but acclimatise less well. We do not recommend smoking as a preventive measure for AMS but highlight the effects of smoking on $\mathrm{NO}$ metabolism and the 
potential roles for $\mathrm{CO}$, nicotine or other active compounds found in cigarette smoke in adaptation to altitude.

\section{DISCLOSURE}

Since it is well documented that the tobacco industry has been manipulating science, scientists and the general public for decades, the present authors declare that none of them has or has ever had any ties to the tobacco industry and that this study is independent from any financial or other influence from the tobacco industry.

Contributors TYW conceived the study, analysed the data and participated in writing; SOD, JLL, JHJ, ZCC, RCD, JZZ and ODT collected and analysed data; BK participated in data analysis, interpretation of the results and writing the final manuscript.

Funding This study was supported by competitive grants NNSF-30393130, 973-2012CB518202 and EAF XZ-101, People's Republic China.

Competing interests None.

Ethics approval China National Science Foundation (NNSF) and the Qinghai High Altitude Medical Research Institute Committee on Human Research.

Provenance and peer review Not commissioned; externally peer reviewed.

Data sharing statement Any interested scholars can ask the corresponding author for an access to the original data.

\section{REFERENCES}

1. GATS. Global Adult Tobacco Survey (GATS)—China Fact Sheet 2010. http://www. who.int/tobacco/surveillance/en tfi china gats factsheet 2010.pdf

2. Hultgren HN. High Altitude Medicine. Stanford: Hultgren Publications, 1997.

3. MacLean N. Smoking and acclimatisation to altitude. Br Med J 1979;2:799.

4. Roach RC, Bärtsch P, Oelz 0, et al. The Lake Louise Acute Mountain Sickness Scoring System. Hypoxia and Molecular Medicine. Burlington, Vermont: Queen City Press, 1993:272-4.

5. Gaillard S, Dellasanta P, Loutan L, et al. Awareness, prevalence, medication use, and risk factors of acute mountain sickness in tourists trekking around the Annapurnas in Nepal: a 12-year follow-up. High Alt Med Biol 2004;5:410-19.

6. Schneider M, Bernasch D, Weymann J, et al. Acute mountain sickness: influence of susceptibility, preexposure, and ascent rate. Med Sci Sports Exerc 2002;34:1886-91.

7. Richalet JP, Larmignat $P$, Poitrine $E$, et al. Physiological risk factors of severe high altitude illness: a prospective cohort study. Am J Respir Crit Care Med 2012;185:192-8.

8. Lindgarde $\mathbf{F}$, Lilljekvist R. Failure of long-term acclimatization in smokers moving to high altitude. Acta Med Scand 1984;216:317-22.

9. Hackett PH, Roach RC. High-altitude illness. N Engl J Med 2001;345:107-14.

10. Wilson $\mathbf{M H}$, Imray $\mathbf{C H}$, Hargens AR. The headache of high altitude and microgravity - similarities with clinical syndromes of cerebral venous hypertension. High Alt Med Biol 2011:12:379-86.

11. Gupta S, Nahas SJ, Peterlin BL. Chemical mediators of migraine: preclinical and clinical observations. Headache 2011:51:1029-45.
12. Mazzuero G, Mazzuero A, Pascariello A. Severe acute mountain sickness and suspect high altitude cerebral edema related to nitroglycerin use. High Alt Med Biol 2008;9:241-3.

13. Ghofrani HA, Reichenberger F, Kohstall MG, et al. Sildenafil increased exercise capacity during hypoxia at low altitudes and at Mount Everest base camp: a randomized, double-blind, placebo-controlled crossover trial. Ann Intern Med 2004;141:169-77.

14. Toda $\mathbf{N}$, Toda $\mathrm{H}$. Nitric oxide-mediated blood flow regulation as affected by smoking and nicotine. Eur J Pharmacol 2010;649:1-13.

15. Malinovschi A, Janson C, Holmkvist T, et al. Effect of smoking on exhaled nitric oxide and flow-independent nitric oxide exchange parameters. Eur Respir $\mathrm{J}$ 2006;28:339-45.

16. Bloch KE, Latshang TD, Turk AJ, et al. Nocturnal periodic breathing during acclimatization at very high altitude at Mount Muztagh Ata (7,546 m). Am J Resp Crit Care 2010:182:562-8.

17. Prabhakar NR. NO and CO as second messengers in oxygen sensing in the carotid body. Respir Physiol 1999:115:161-8.

18. Argacha JF, Xhaet 0 , Gujic $\mathrm{M}$, et al. Nicotine increases chemoreflex sensitivity to hypoxia in non-smokers. J Hypertens 2008;26:284-94.

19. Wright JL, Levy RD, Churg A. Pulmonary hypertension in chronic obstructive pulmonary disease: current theories of pathogenesis and their implications for treatment. Thorax 2005;60:605-9.

20. Moudgil R, Michelakis ED, Archer SL. Hypoxic pulmonary vasoconstriction. J App Physiol 2005;98:390-403.

21. Scherrer U, Rexhaj E, Jayet PY, et al. New insights in the pathogenesis of highaltitude pulmonary edema. Prog Cardiovasc Dis 2010;52:485-92.

22. Joseph V, Pequignot JM. Breathing at high altitude. Cell Mol Life Sci 2009:66:3565-73.

23. Leon-Velarde F, Villafuerte FC, Richalet JP. Chronic mountain sickness and the heart. Prog Cardiovasc Dis 2010;52:540-9.

24. Pei SX, Chen XJ, Si Ren BZ, et al. Chronic mountain sickness in Tibet. $0 \mathrm{~J}$ Med 1989;71:555-74

25. Tremper KK. Pulse oximetry. Chest 1989;95:713-15.

26. Brewer GJ, Eaton JW, Grover RF, et al. Cigarette smoking as a cause of hypoxemia in man at altitude. Chest 1971;59:Suppl: 30S+.

27. Basu CK, Selvamurthy W, Bhaumick G, et al. Respiratory changes during initial days of acclimatization to increasing altitudes. Aviat Space Environ Med 1996;67:40-5

28. Pellegrino R, Pompilio P, Quaranta $\mathrm{M}$, et al. Airway responses to methacholine and exercise at high altitude in healthy lowlanders. J Appl Physiol 2010;108:256-65.

29. Gautier H, Peslin R, Grassino A, et al. Mechanical properties of the lungs during acclimatization to altitude. J App/ Physiol 1982;52:1407-15

30. Pollard AJ, Mason NP, Barry PW, et al. Effect of altitude on spirometric parameters and the performance of peak flow meters. Thorax 1996:51:175-8.

31. Mason NP, Barry PW, Pollard AJ, et al. Serial changes in spirometry during an ascent to 5,300 m in the Nepalese Himalayas. High Alt Med Biol 2000; 1:185-95.

32. Girdler SS, Maixner W, Naftel HA, et al. Cigarette smoking, stress-induced analgesia and pain perception in men and women. Pain 2005:114:372-85.

33. Edwards R. The problem of tobacco smoking. Br Med J 2004;328:217-19.

34. Ervasti 0, Juopperi K, Kettunen $P$, et al. The occurrence of frostbite and its risk factors in young men. Int J Circumpolar Health 2004;63:71-80.

35. Forte VA, Muza SR, Fulco CS, et al. Smoking accentuates the decrement in maximal oxygen uptake at high altitude. FASEB J 1996;10:3723. 